\title{
DIFFERENTIAL EXPRESSION PATTERN OF GENES INVOLVED IN OXYGEN METABOLISM IN EPITHELIAL OVIDUCTAL CELLS DURING PRIMARY IN VITRO CULTURE
}

Katarzyna Stefańska ${ }^{1}$, Sandra Knap ${ }^{1}$, Magdalena Kulus ${ }^{2}$, Ievgenia Kocherova ${ }^{3}$, Piotr Celichowski ${ }^{1}$, Michal Jeseta ${ }^{4}$, Marie Machatkova ${ }^{5}$, Dorota Bukowska ${ }^{2}$, Paweł Antosik ${ }^{2}$

\begin{abstract}
Oxygen metabolism is crucial in establishing successful pregnancy, since excessive amount of reactive oxygen species (ROS) may exert deleterious effects on the developing embryo. There are several defense mechanisms against oxidative stress in the female reproductive tract, including production of antioxidant enzymes by oviductal epithelial cells (OECs). Undoubtedly, OECs play major part in female fertility and may also serve as an in vitro model of the oviduct. Therefore, the aim of this study was to investigate the expression of genes involved in oxygen metabolism. We have isolated OECs from oviducts of crossbred gilts $(n=45)$ and maintained their in vitro culture for 30 days, collecting their RNA at days 1, 7, 15 and 30. The gene expression was determined with the use of Affymetrix® Porcine Gene 1.1 ST Array Strip. Our results revealed 166 differentially expressed genes belonging to four ontology groups: „cellular response to oxidative stress", "cellular response to oxygen-containing compound", "cellular response to oxygen levels" and "cellular response to reactive oxygen species", most of which are also involved in other major processes in the organism. However, our findings provide a valuable insight into porcine reproductive biology and may be utilized in optimization of assisted reproduction techniques.
\end{abstract}

Running title: Genes involved in oxygen metabolism in oviductal epithelial cells

Keywords: oviductal epithelial cells, culture, oxygen metabolism, transcriptom

\footnotetext{
${ }^{1}$ Department of Histology and Embryology, Poznan University of Medical Sciences, Poznan, Poland

${ }^{2}$ Veterinary Centre, Nicolaus Copernicus University in Torun, Torun, Poland

${ }^{3}$ Department of Anatomy, Poznan University of Medical Sciences, Poznan, Poland

${ }^{4}$ Department of Obstetrics and Gynaecology, University Hospital and Masaryk University, Brno, Czech Republic

${ }^{5}$ Veterinary Research Institute, Brno, Czech Republic

* Correspondence: pantosik@umk.pl

Full list of author information is available at the end of article
} 


\section{Introduction}

The oviduct is a part of mammalian female reproductive tract, connecting the ovary to the uterus, that plays an invaluable role in female fertility. It is involved in gamete and embryo transport, supports fertilization and provides an optimal environment for gamete survival and preimplantation embryo development. The oviductal epithelium consists of two types of epithelial cells: ciliated and non-ciliated secretory cells, the latter producing components of oviductal fluid, which participates in gamete guidance and embryo cleavage [1].

Oxygen metabolism is particularly important in establishing a successful pregnancy, with both gametes and embryos producing reactive oxygen species (ROS). Porcine preimplantation embryos produce ROS via oxidative phosphorylation and inhibition of this process results in better outcome in in vitro development. Apart from that, there are several exogenous factors that can enhance ROS generation by embryos. However, the excessive presence of ROS may exert deleterious effects on embryos via lipid, protein and nucleic acid alterations, which can result in mitochondrial disfunction, embryo cell block, lack of ATP and apoptosis [2].

Taking everything into consideration, there is no doubt that defense mechanisms against oxidative stress must be employed to ensure gamete and embryo survival and proper development. Except for the antioxidant enzymes stored as mRNA in the oocyte, there is also an external protection through the oviductal environment. Several antioxidant enzymes, such as glutathione peroxidase, $\mathrm{Cu}-\mathrm{Zn}$-superoxide dismutase, or catalase, have been found to be expressed in oviducts of humans and mice [3]. Moreover, oviductal epithelial cell (OECs) membrane proteins bind to the human spermatozoa, which results in its protection against oxidative stress, as indicated by Huang et al. [4].

OEC in vitro culture may serve as a model of the oviduct and be helpful in elucidating complex events taking place in this part of the female reproductive tract. A deeper understanding of the porcine reproductive biology is of great importance, since the pig is one of the most popular meat sources, with its anatomy and physiology resembling those of humans [5]. Therefore, in this study, we established a long-term primary culture of porcine OECs and performed microarray analyses at specific time periods, to determine differentially expressed genes involved in oxygen metabolism.

\section{Material and Methods Animals}

In this study, crossbred gilts $(n=45)$ at the age of about nine months and which displayed at least two regular estrous cycles were collected from a commercial herd. All the animals were checked daily for estrus behavior and were slaughtered after reach- ing the anestrus phase of the estrus cycle. The uteri were then transported to the laboratory within 30 $\min$ at $38^{\circ} \mathrm{C}$.

\section{Oviductal epithelial cells (OECs) selection and culture}

Oviducts were washed twice in Dulbecco's phosphate buffered saline (PBS) (137 mM NaCl, $27 \mathrm{mM}$ $\mathrm{KCl}, 10$ mM Na2HPO4, 2 mM KH2PO4, pH 7.4). Epithelial cells were removed using sterile surgical blades. Then, the epithelium was incubated with collagenase I (Sigma Aldrich, Madison, USA), 1mg/ $\mathrm{mL}$ in Dubecco's modified Eagle's medium (DMEM; Sigma Aldrich, Madison, USA) for $1 \mathrm{~h}$ at 37oC. The cell suspension obtained from this digestion was filtered through $40 \mu \mathrm{m}$ pore size strainer to remove blood and single cells. The residue was collected by rinsing the strainer with DMEM. The cells were then centrifuged ( $200 \mathrm{x} \mathrm{g}, 10 \mathrm{~min}$.). Next, they were washed in PBS and centrifuged again. Later, they were incubated with $0.5 \%$ Trypsin/EDTA (Sigma Aldrich, Madison, USA) at $37 \mathrm{oC}$ for $10 \mathrm{~min}$. The reaction was stopped with fetal calf serum (FCS; Sigma Aldrich, Madison, USA). After incubation, the cells were filtered and centrifuged for the last time. The final cell pellet was suspended in DMEM, supplemented with $10 \% \mathrm{FCS}, 100 \mathrm{U} / \mathrm{mL}$ penicillin, $100 \mu \mathrm{g} / \mathrm{mL}$ streptomycin and $1 \mu \mathrm{g} / \mathrm{mL}$ amphotericin B. The cells were cultured at $37^{\circ} \mathrm{C}$ in a humidified atmosphere of $5 \% \mathrm{CO} 2$. Once the OEC cultures attained $70-80 \%$ confluency, they were passaged by washing with PBS, digested with $0.025 \%$ Trypsin/ EDTA, neutralized by a $0.0125 \%$ trypsin inhibitor (Cascade Biologics, Portland, USA), centrifuged, and resuspended at a seeding density of $2 * 10^{4}$ cells / $\mathrm{cm} 2$. The culture medium was changed every three days. The culture was maintained for30 days.

\section{RNA extraction from oviductal epithelial cells (OECs)}

Oviductal epithelial cell were pooled and harvested 24h, 7 days, 15 days and 30 days after the beginning of culture. Total RNA was extracted from the samples using TRI Reagent (Sigma, St Louis, MO, USA) and RNeasy MinElute cleanup Kit (Qiagen, Hilden, Germany). The total mRNA amount was determined from the optical density at $260 \mathrm{~nm}$, and the RNA purity was estimated using the $260 / 280 \mathrm{~nm}$ absorption ratio (higher than 1.8) (NanoDrop spectrophotometer, Thermo Scientific, ALAB, Poland). The RNA integrity and quality were checked on a Bioanalyzer 2100 (Agilent Technologies, Inc., Santa Clara, CA, USA). The resulting RNA integrity numbers (RINs) were between 8.5 and 10 with an average of 9.2 (Agilent Technologies, Inc., Santa Clara, CA, USA). The RNA in each sample was diluted to a concentration of $100 \mathrm{ng} / \mu \mathrm{l}$ with an OD260/OD280 ratio of 1.8/2.0. From each RNA sample, 100 ng of RNA was taken for microarray expression assays. 


\section{Microarray expression analysis and statistics}

Total RNA (100 ng) from each pooled sample was subjected to two rounds of sense cDNA amplification (Ambion ${ }^{\circledR}$ WT Expression Kit). The obtained cDNA was used for biotin labeling and fragmentation usingAffymetrix GeneChip ${ }^{\circledR}$ WT Terminal Labeling and Hybridization (Affymetrix, Santa Clara, CA, USA). Biotin-labeled fragments of cDNA (5.5 $\mu \mathrm{g})$ were hybridized to the Affymetrix ${ }^{\circledR}$ Porcine Gene 1.1 ST Array Strip $\left(48^{\circ} \mathrm{C} / 20 \mathrm{~h}\right)$. Microarrays were then washed and stained, according to the technical protocol, using the Affymetrix GeneAtlas Fluidics Station. The array strips were scanned employing the Imaging Station of the GeneAtlas System. Preliminary analysis of the scanned chips was performed using Affymetrix GeneAtlas ${ }^{\mathrm{TM}}$ Operating Software. The quality of gene expression data was confirmed according to the quality control criteria provided by the software. The obtained CEL files were imported into downstream data analysis software.

All of the presented analyses and graphs were compiled using Bioconductor and R programming languages. Each CEL file was merged with a description file. To correct background, normalize, and summarize results, we used the Robust Multiarray Averaging (RMA) algorithm. To determine the statistical significance of the analyzed genes, moderated t-statistics from the empirical Bayes method were performed. The obtained p-value was corrected for multiple comparisons using Benjamini and Hochberg's false discovery rate. Selection of significantly altered genes was based on a p-value beneath 0.05 and expression higher than two-fold.

Differentially expressed genes were subjected selection by examination of genes involved in oxygen metabolism. The differentially expressed gene list (separated for up- and down-regulated genes) was uploaded to the DAVID software (Database for Annotation, Visualization and Integrated Discovery) [6], where genes belonging to the terms of all four Gene Ontologies (GOs) of interest were extracted. Expression data of these genes was also subjected to a hierarchical clusterization procedure, with their expression values presented as a heat map.

Subsequently, we analyzed the relation between the genes belonging to the chosen GO terms using the GO plot package [7]. The Go plot package had calculated the z-score: the number of up-regulated genes minus the number of down- regulated genes divided by the square root of the count. This information allowed to estimate the change course of each gene-ontology term.

Interactions between differentially expressed genes/proteins belonging to the studied gene ontology groups were investigated by the STRING10 software (Search Tool for the Retrieval of Interacting Genes) [8]. The list of gene names was used as a query for interaction prediction. The search criteria were based on co-occurrences of genes/proteins in scientific texts (text mining), co-expression, and experimentally observed interactions. The results of such analyses generated a gene/protein interaction network where the intensity of the edges reflected the strength of the interaction score.

Finally, the functional interactions between genes that belongs to the chosen GO BP terms were investigated by the REACTOME FIViz application to the Cytoscape 3.6.0 software. The Reactome FIViz app is designed to find pathways and network patterns related to cancer and other types of diseases. This app accesses the pathways stored in the Reactome database, allowing to perform pathway enrichment analysis for a set of genes, visualize hit pathways using manually laid-out pathway diagrams directly in Cytoscape, and investigate functional relationships among genes in hit pathways. The app can also access the Reactome Functional Interaction (FI) network, a highly reliable, manually curated pathway-based protein functional interaction network covering over $60 \%$ of human proteins.

\section{Ethical approval}

The research related to animal use has been complied with all the relevant national regulations and instructional policies for the care and use of animals. Bioethical Committee approval no. 32/2012.

\section{Results}

Whole transcriptome profiling with Affymetrix microarrays allows us to analyze the gene expression changes between 7, 15 and 30 days of porcine oviductal epithelial cell culture. Using Affymetrix® Porcine Gene 1.1 ST Array Strip, we have examined the expression of 12257 transcripts. Genes with fold change higher than abs (2) and with corrected p-value lower than 0.05 were considered as differentially expressed. This set of genes consists of 2533 different transcripts.

DAVID (Database for Annotation, Visualization and Integrated Discovery) software was used for extraction of gene ontology biological process terms (GO BP) that contain differently expressed transcripts. Up and down regulated gene sets were subjected to the DAVID search separately and only gene sets with adj. p-value lower than 0.05 were selected. The DAVID software analysis showed that the differently expressed genes belonged to 657 Gene ontology terms. In this paper, we focused on 166 genes that belong to "cellular response to oxidative stress", "cellular response to oxygen-containing compound", "cellular response to oxygen levels" and "cellular response to reactive oxygen species" GO BP terms. These sets of genes were subjected to hierarchical clusterization procedure and presented as heatmaps (Fig. 1). The gene symbols, fold changes in expression, Entrez gene IDs and corrected p-values of these genes were shown in table 1.

The enrichment of each GO BP term was calculated as a z-score and shown on the circle diagram (Fig. 2). 


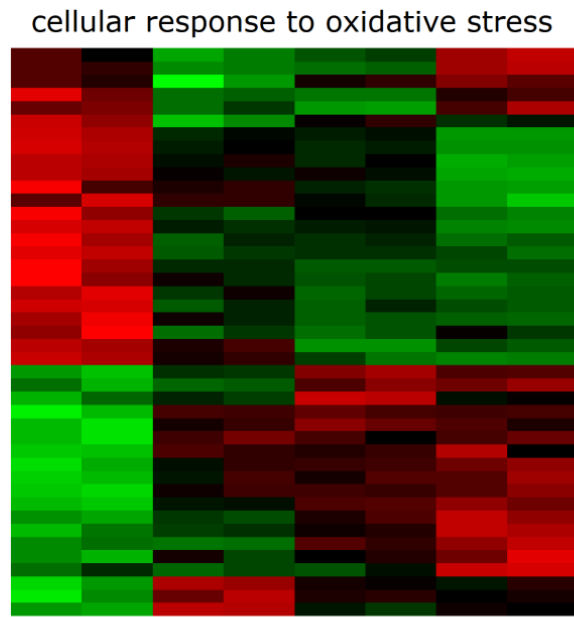

ธ ธ

cellular response to oxygen-containing compound

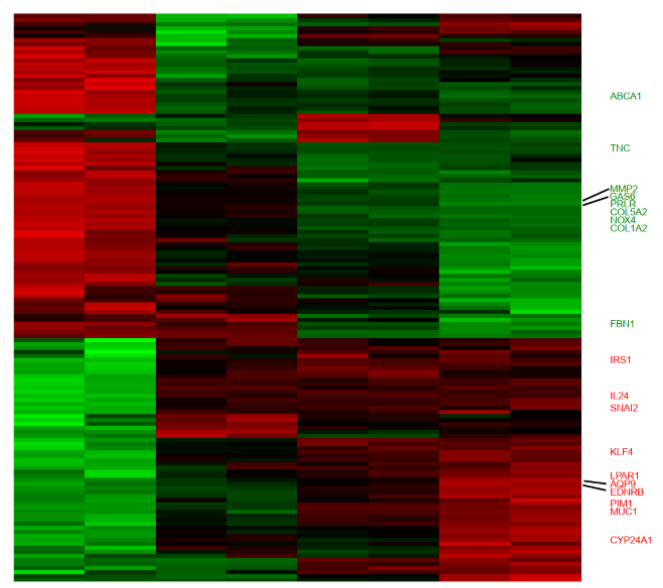

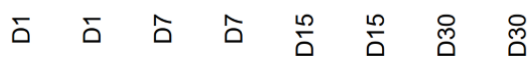

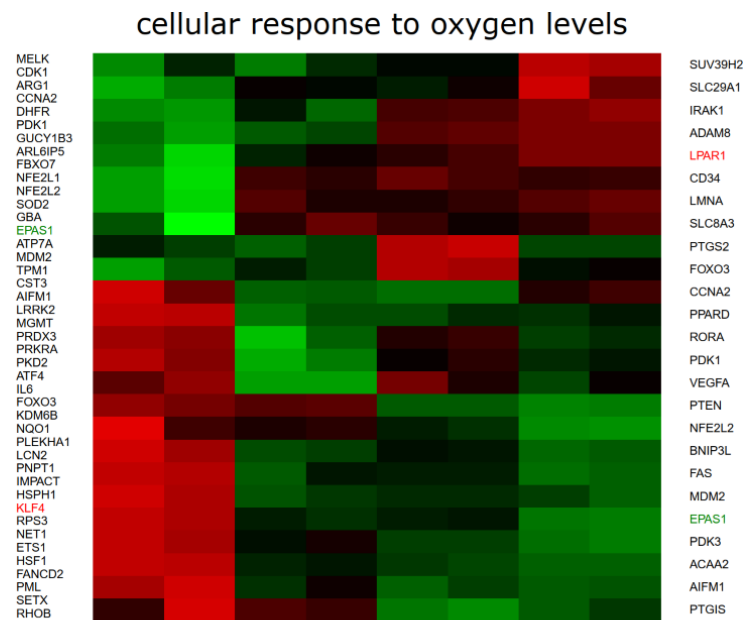

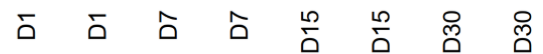

cellular response to reactive oxygen species

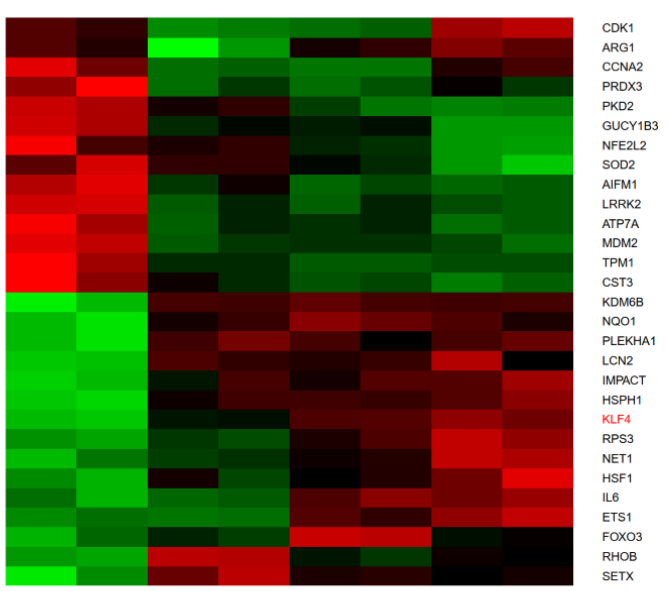

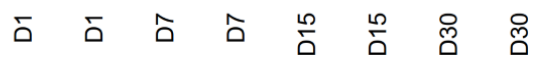

FIGURE 1 Heat map representation of differentially expressed genes belonging to the to "cellular response to oxidative stress", "cellular response to oxygen-containing compound", "cellular response to oxygen levels" and "cellular response to reactive oxygen species" GO BP terms. Arbitrary signal intensity acquired from microarray analysis is represented by colours (green, higher; red, lower expression). Log2 signal intensity values for any single gene were resized to Row Z-Score scale (from -2, the lowest expression to +2 , the highest expression for single gene). The 20 genes selected to this publication was marked green for upregulation and red for downregulation

The chosen GO BP terms contain 166 differently expressed genes. Therefore, we calculated the mean fold change ratio value of each gene between 7,15 and 30 days of culture. Based on that criteria, we chose the 10 most downregulated and 10 most upregulated genes for further analysis.

In Gene Ontology database, genes that form one particular GO can also belong to other GO term categories. For this reason, we explored the gene intersections between the selected GO BP terms. The relation between those GO BP terms was presented as circle plot (Fig. 3) as well as heatmap (Fig. 4).

STRING interaction network was generated among the differentially expressed genes belonging to each of the selected GO BP terms. Using such prediction method provided us with a molecular interaction network formed between protein products of the studied genes (Fig. 5). Finally, we investigated the functional interactions between the chosen genes with REACTOME FIViz app to the Cytoscape 3.6.0 software. The results were shown in (Fig. 6).

\section{Discussion}

Oviductal epithelial cells are known for their crucial role in providing an optimal environment for gamete survival, supporting fertilization and early embryo development [1]. Therefore, the aim of this study was to gain a better insight into molecular events that influence these cells' functioning. We utilized a microarray approach to determine differentially expressed genes in primary long-term in vitro culture of porcine OECs, with our results revealing alteration in levels of 2533 transcripts. In this paper, we focused on 166 genes belonging to 
TABLE 1 Gene symbols, fold change in expression ratio, Entrez gene IDs, corrected p values and mean value of fold change ratio of studied genes

\begin{tabular}{c|c|c|c|c|c|c|c}
\hline $\begin{array}{c}\text { GENE } \\
\text { SYMBOL }\end{array}$ & $\begin{array}{c}\text { RATI0 } \\
\text { D7/D1 }\end{array}$ & $\begin{array}{c}\text { RATIO } \\
\text { D15/D1 }\end{array}$ & $\begin{array}{c}\text { RATIO } \\
\text { D30/D1 }\end{array}$ & $\begin{array}{c}\text { ADJUSTED } \\
\text { P.VALUE } \\
\text { D7/D1 }\end{array}$ & $\begin{array}{c}\text { ADJUSTED } \\
\text { P.VALUE } \\
\text { D15/D1 }\end{array}$ & $\begin{array}{c}\text { ADJUSTED } \\
\text { P.VALUE } \\
\text { D30/D1 }\end{array}$ & $\begin{array}{c}\text { MEAN } \\
\text { RATIO }\end{array}$ \\
\hline $\boldsymbol{A Q P 9}$ & $-4,009$ & $-7,510$ & $-69,247$ & $2,57 \mathrm{E}-04$ & $2,80 \mathrm{E}-05$ & $5,88 \mathrm{E}-07$ & $-26,922$ \\
\hline $\boldsymbol{M U C 1}$ & $-4,154$ & $-28,786$ & $-47,147$ & $4,67 \mathrm{E}-05$ & $9,76 \mathrm{E}-07$ & $2,44 \mathrm{E}-07$ & $-26,695$ \\
\hline $\boldsymbol{E D N R B}$ & $-2,719$ & $-11,232$ & $-53,971$ & $7,06 \mathrm{E}-04$ & $6,74 \mathrm{E}-06$ & $4,60 \mathrm{E}-07$ & $-22,641$ \\
\hline $\boldsymbol{I L 2 4}$ & $-11,995$ & $-16,079$ & $-14,006$ & $9,50 \mathrm{E}-06$ & $3,21 \mathrm{E}-06$ & $1,76 \mathrm{E}-06$ & $-14,027$ \\
\hline $\boldsymbol{C Y P 2 4 A 1}$ & $-6,704$ & $-3,398$ & $-25,263$ & $2,69 \mathrm{E}-05$ & $1,36 \mathrm{E}-04$ & $7,91 \mathrm{E}-07$ & $-11,788$ \\
\hline $\boldsymbol{K L F 4}$ & $-4,866$ & $-11,123$ & $-17,404$ & $4,71 \mathrm{E}-05$ & $3,93 \mathrm{E}-06$ & $1,01 \mathrm{E}-06$ & $-11,131$ \\
\hline $\boldsymbol{P I M 1}$ & $-2,278$ & $-7,702$ & $-14,754$ & $8,42 \mathrm{E}-04$ & $7,24 \mathrm{E}-06$ & $1,14 \mathrm{E}-06$ & $-8,245$ \\
\hline SNAI2 & $-5,442$ & $-7,434$ & $-7,688$ & $4,11 \mathrm{E}-04$ & $1,29 \mathrm{E}-04$ & $7,85 \mathrm{E}-05$ & $-6,854$ \\
\hline LPAR1 & $-2,576$ & $-3,932$ & $-5,953$ & $4,96 \mathrm{E}-03$ & $6,50 \mathrm{E}-04$ & $1,17 \mathrm{E}-04$ & $-4,154$ \\
\hline IRS1 & $-3,201$ & $-4,110$ & $-4,254$ & $6,44 \mathrm{E}-05$ & $1,52 \mathrm{E}-05$ & $8,37 \mathrm{E}-06$ & $-3,855$ \\
\hline $\boldsymbol{A B C A 1}$ & 6,313 & 5,557 & 10,336 & $3,95 \mathrm{E}-05$ & $3,12 \mathrm{E}-05$ & $4,36 \mathrm{E}-06$ & 7,402 \\
\hline $\boldsymbol{T N C}$ & 6,573 & 8,352 & 8,773 & $1,83 \mathrm{E}-05$ & $4,91 \mathrm{E}-06$ & $2,42 \mathrm{E}-06$ & 7,899 \\
\hline $\boldsymbol{G A S 6}$ & 4,336 & 9,495 & 16,755 & $1,66 \mathrm{E}-05$ & $1,63 \mathrm{E}-06$ & $2,93 \mathrm{E}-07$ & 10,195 \\
\hline $\boldsymbol{E P A S 1}$ & 7,630 & 6,655 & 16,828 & $8,57 \mathrm{E}-06$ & $5,17 \mathrm{E}-06$ & $5,84 \mathrm{E}-07$ & 10,371 \\
\hline $\boldsymbol{M M P 2}$ & 5,014 & 11,115 & 19,107 & $1,73 \mathrm{E}-05$ & $1,68 \mathrm{E}-06$ & $3,42 \mathrm{E}-07$ & 11,745 \\
\hline $\boldsymbol{C O L 5 A 2}$ & 5,878 & 20,359 & 24,539 & $7,45 \mathrm{E}-06$ & $7,45 \mathrm{E}-07$ & $2,31 \mathrm{E}-07$ & 16,925 \\
\hline $\boldsymbol{N O X 4}$ & 11,056 & 21,128 & 32,071 & $4,90 \mathrm{E}-06$ & $9,73 \mathrm{E}-07$ & $2,31 \mathrm{E}-07$ & 21,418 \\
\hline $\boldsymbol{F B N 1}$ & 3,692 & 27,547 & 62,849 & $1,51 \mathrm{E}-04$ & $1,63 \mathrm{E}-06$ & $3,07 \mathrm{E}-07$ & 31,363 \\
\hline $\boldsymbol{P R L R}$ & 8,169 & 22,945 & 73,134 & $7,45 \mathrm{E}-06$ & $9,73 \mathrm{E}-07$ & $2,31 \mathrm{E}-07$ & 34,749 \\
\hline $\boldsymbol{C O L 1 A 2}$ & 15,573 & 56,376 & 61,329 & $3,63 \mathrm{E}-06$ & $7,45 \mathrm{E}-07$ & $2,31 \mathrm{E}-07$ & 44,426 \\
\hline
\end{tabular}

7D

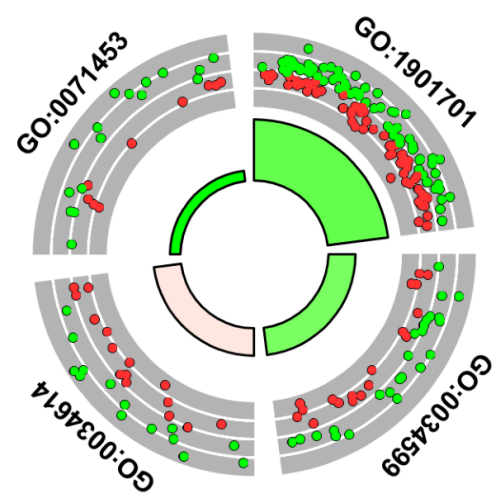

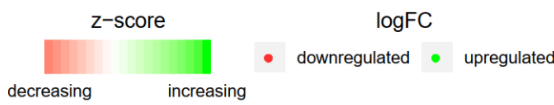

15D

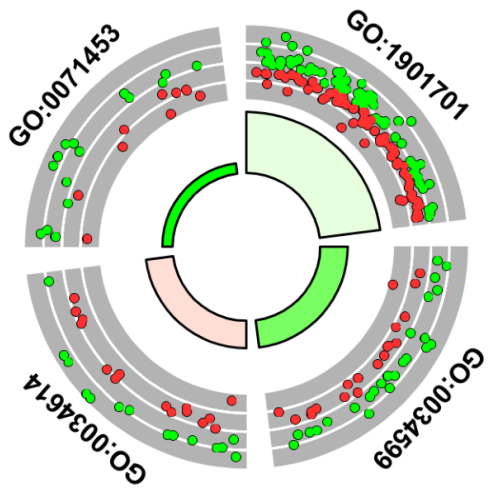

$30 \mathrm{D}$

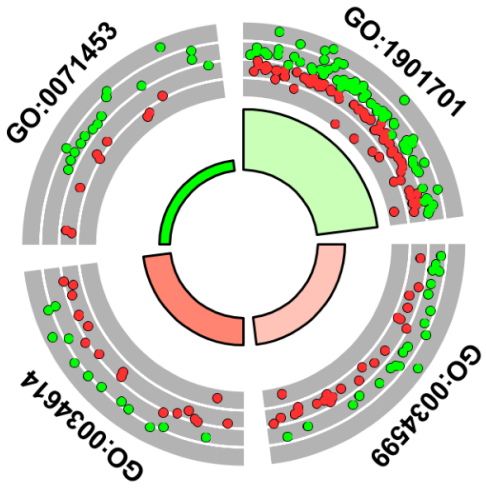

ID GO:1901701 cellular response to oxygen-containing compound GO:0034599 $\mathrm{GO}: 0034614$ GO:0071453 cellular response to oxidative stress cellular response to reactive oxygen species cellular response to oxygen levels

FIGURE 2 The circle plot showing the differently expressed genes and z-score of the "cellular response to oxidative stress", "cellular response to oxygen-containing compound", "cellular response to oxygen levels" and "cellular response to reactive oxygen species" GO BP terms. The outer circle shows a scatter plot for each term of the fold change of the assigned genes. Green circles display up- regulation and red ones down- regulation. The inner circle shows the z-score of each GO BP term. The width of the each bar corresponds to the number of genes within GO BP term and the color corresponds to the z-score 


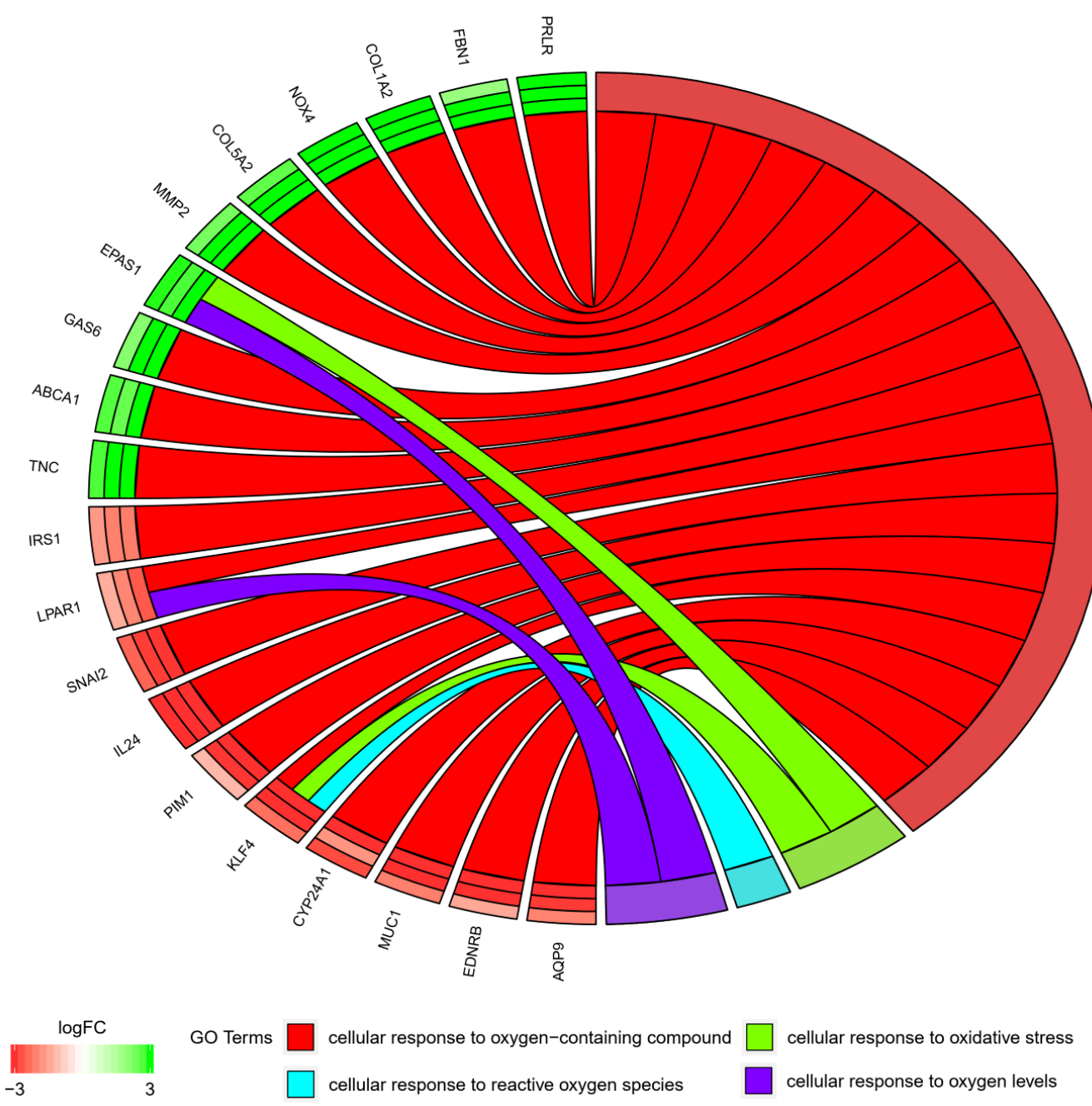

FIGURE 3 The representation of the mutual relationship of differently expressed genes that belongs to 20 chosen genes from "cellular response to oxidative stress", "cellular response to oxygen-containing compound", "cellular response to oxygen levels" and "cellular response to reactive oxygen species" GO BP terms. The ribbons indicate which gene belongs to which categories. The middle circle represents logarithm from fold change (LogFC) between D7/D1, D15/D1 and D30/ D1 respectively. The color of each block corresponds to the LogFC of each gene (green - upregulated, red - downregulated). The genes were sorted by logFC from most to least changed gene

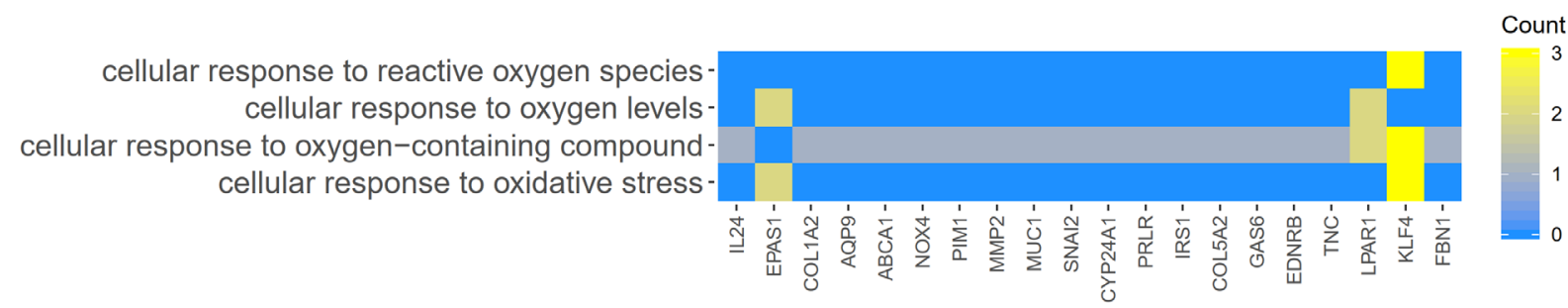

FIGURE. 4 Heatmap showing the gene occurrence between chosen 20 differently expressed genes that belongs to "cellular response to oxidative stress", "cellular response to oxygen-containing compound", "cellular response to oxygen levels" and "cellular response to reactive oxygen species" GO BP terms. The yellow color is associated with gene occurrence in the GO Term. The intensity of the color is corresponding to amount of GO BP terms that each gene belongs to

four ontology groups: „cellular response to oxidative stress", "cellular response to oxygen-containing compound", "cellular response to oxygen levels and cellular response to reactive oxygen species".
The group of ten most downregulated genes includes: AQP9, MUC1, EDNRB, IL24, CYP24A1, KLF4, PIM1, SNAI2, LPAR1 and IRS1. AQP9 encodes aquaporin 9 and, in the course of this study, exhibited the 


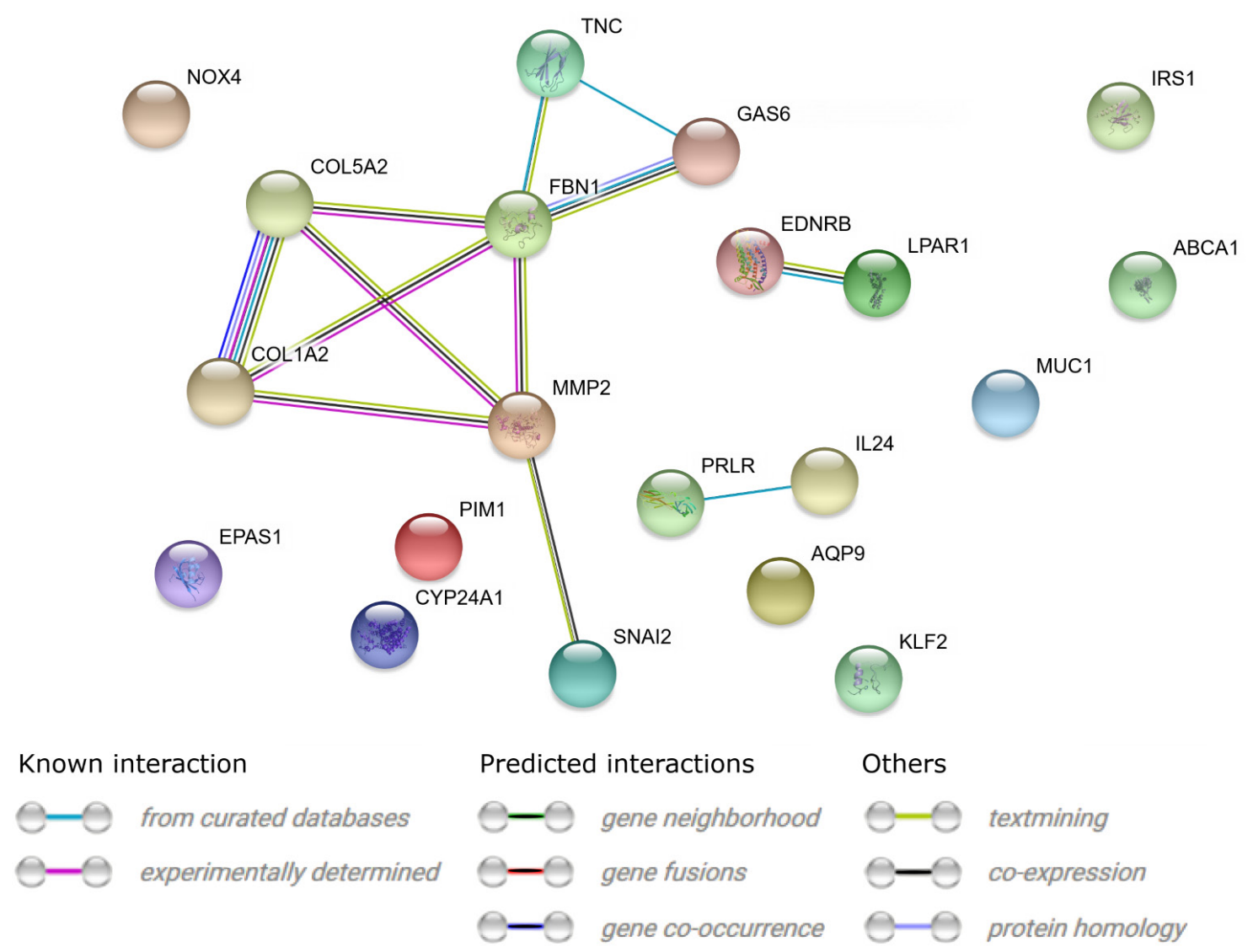

FIGURE 5 STRING-generated interaction occurrence between 20 chosen differently expressed genes that belongs to the "cellular response to oxidative stress", "cellular response to oxygen-containing compound", "cellular response to oxygen levels" and "cellular response to reactive oxygen species" GO BP terms. The intensity of the edges reflects the strength of interaction score

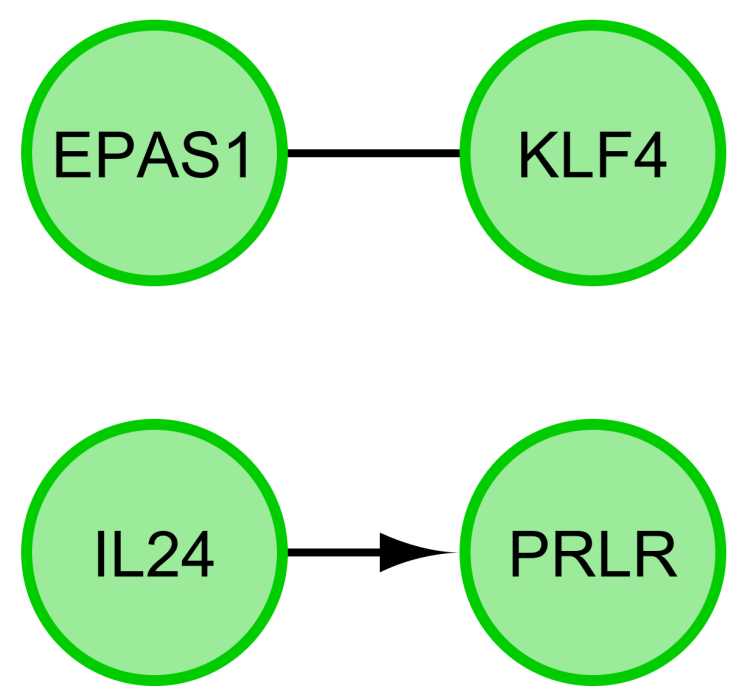

FIGURE 6 Functional interaction (FI) between 20 chosen differently expressed genes that belongs to the "cellular response to oxidative stress", "cellular response to oxygen-containing compound", "cellular response to oxygen levels" and "cellular response to reactive oxygen species" GO BP terms. In following figure "->" stands for activating/catalyzing, "-|" for inhibition, "-" for FIs extracted from complexes or inputs, and "---" for predicted FIs

most significant decrease in expression. The family of aquaporins consists of membrane channels that are water-selective and enable water transport through the cell membrane. The presence of AQP9 in porcine OECs has already been demonstrated by Skowronski et al., with its role in epithelial fluid movement in the oviduct suggested [9]. Subsequent studies conducted by the same author also revealed 
that AQP9 expression reached the highest level on days 2-4 and 18-20 of the oestrous cycle, thus it is dependent on steroid hormone levels [10].

MUC1 encodes the transmembrane mucin 1, which is typically expressed on the apical borders of secretory epithelial cells. The study by Lin et al. indicated its role in response to oxidative stress, since its expression is upregulated in such conditions [11]. MUC1 presence has been detected in human fallopian tube epithelium [12], whereas our results show its downregulation in porcine OECs after long term in vitro cultivation.

Another downregulated gene, EDNRB, encodes type B receptor for endothelin. Endothelins exert diverse functions throughout the organism, including luteolysis, contraction of myometrium and the rupture of the follicle. EDNRB presence has been described in bovine oviductal epithelial cells and smooth muscle [13]. Yamamoto et al. have also suggested this receptor's involvement in oviductal motility. Apart from that, EDNRB is known to interact with LPAR1 (Fig. 5), encoded by another downregulated gene in this study. LPAR1 is a receptor for lysophosphatidic acid (LPA) and is abundant in most human tissues. However, it reaches the highest level of expression in the brain [14]. Abovine study also revealed its presence in oviductal epithelial cells and the role of LPA in fertilization, as well as in gamete and embryo transport through an influence on prostaglandin production [15].

IL24 encodes interleukin 24, a cytokine expressed in the tissues of immune system, such as the thymus, spleen, peripheral blood leukocytes and melanocytes, involved in immune regulation, wound healing and protection against bacterial infections. However, at supra-physiological levels, it exerts anti-cancer properties, such as apoptosis induction, inhibition of invasion or autophagy [16]. Moreover, IL-24-mediated apoptosis is limited to cancer cells without affecting the normal ones, with this process dependent on ER stress induction and ROS production [17]. Our team, on the other hand, observed a significant decrease in this gene's expression after porcine OEC long term in vitro cultivation.

Another downregulated gene, $C Y P 24 A 1$, encodes an enzyme involved in catabolism of metabolically active vitamin D. Its expression has already been described in human OECs, both ciliated and secretory. This protein's level is cycle-dependent, which suggests a tight control of active vitamin $D$ in the fallopian tube [18]. A study performed on human preeclamptic placentas also revealed a link between hypoxia and CYP24A1 upregulation. Therefore, oxidative stress occurring during pregnancy may affect vitamin D metabolism and subsequently increase the risk of preeclampsia [19].

SNAI2 and KLF4, both belonging to zinc finger transcription factor family, showed a significant decrease in their expression after long term porcine
OEC cultivation. SNAI2 belongs to an evolutionary conserved family of transcription factors and plays crucial role in mesoderm formation, embryonic development and epithelial-mesenchymal transition (EMT). Apart from that, its overexpression has been linked to re-epithelialization [20]. Moreover, SNAI2 expression is sensitive to hypoxia and oxidative stress and its downregulation has been associated with preeclampsia in humans and rats [21]. KLF4, on the other hand, is engaged in many cellular processes, such as cell growth, proliferation and differentiation and has been indicated as a negative regulator of cell growth in e.g. gut epithelium [22]. However, this is probably not the case in porcine OECs, since we observed its downregulation. KLF4 is also known to induce apoptosis via transcriptional activation of BAX and transcriptional repression of BCL-2 in the setting of oxidative stress in granulosa cells [23].

PIM1 encodes a serine/threonine protein kinase engaged in hematopoietic development, with its overexpression occurring in many myeloid and lymphoid acute leukaemias [24]. However, it hasn't been described in porcine OECs yet. Regarding oxidative stress, PIM1 is thought to be upregulated during transient stress and downregulated during chronic stress [25]. The last of ten most downregulated genes, IRS1, encodes insulin receptor substrate 1 , which is phosphorylated after insulin binding to its receptor and participates in insulin signalling pathway [26]. Similarly to PIM1, its presence has not been observed in porcine OECs. However, it is expressed in all cell lineages of peri-implantation mouse embryo, suggesting its important role at this stage of development [27].

Apart from previously described downregulated genes, we have also observed a significant increase in several genes' expression. In this paper we would like to focus on a group of ten most upregulated ones, which consist of: ABCA1, TNC, GAS6, EPAS1, MMP2, COL5A2, NOX4, FBN1, PRLR, COL1A2.

$A B C A 1$, the most upregulated gene in our study, belongs to the family of ATP binding cassette transporters. It is involved in cellular lipid removal pathway via hydrolysation of ATP, which allows substrate transport across the cell membrane [28]. Apart from that, ABCA1 is also thought to participate in apoptotic cell clearance by macrophages, with its expression occurring in variety of human tissues, such as placenta, lung, liver or ovary. Therefore, its role is not limited to reproductive events [29].

In the course of this study we have also observed a significant increase in expression of alpha chains of two types of collagen, namely collagen I and V. Collagens are a group of proteins engaged in maintaining the structural integrity of connective tissue and types I, III and V are usually co-expressed, with their level depending on the cell type [30]. Type I is mostly expressed in skin, bone, tendon and pla- 
centa, whereas little is known about the type $\mathrm{V}$ presence. Based on our results, as well as previous reports by other investigators, both COL5A2 and COL1A2 interact with each other (Fig. 5). A study conducted by Adrikopoulos et al. indicates that type $\mathrm{V}$ collagen is crucial in assembly of collagen molecules into three-dimensional aggregates, since mice with a deletion of Col5a2 gene exhibited symptoms of disorganized type I collagen fibrils [31].

The results obtained in this study indicate that both COL5A2 and COL1A2 interact with FBN1 and MMP2 (Fig. 5), which were also upregulated in porcine OECs after long term cultivation. FBN1 encodes fibrillin 1, which is another protein distributed in the connective tissue, especially in skin, lung, cartilage and tendon. It participates in extracellular microfibril formation [32]. Since it is well established that these genes' mutations underlie the Marfan syndrome, it is obvious that FBN1 exerts widespread functions in the organism. Fibrillin 1 is also known to interact with previously mentioned matrix metalloproteinase 2 , which is involved in extracellular matrix degradation, especially that of type IV collagen a major component of the basement membrane [33]. Apart from that, MMP2 is thought to be involved in Mullerian duct regression and male sexual differentiation, since it acts downstream of AMH (Anti-Mullerian Hormone) and is elevated in male Mullerian duct mesenchyme [34]. Moreover, a study by Wu et al. indicated that MMP2 is expressed in most of the benign and malignant epithelial ovarian tumours and that activated MMP2 may serve as a potential prognostic marker in epithelial ovarian carcinoma [33]. In regards to oxidative stress, a study on hepatic stellate cells revealed that hypoxia enhances MMP2 expression [35] and we conclude that it may also be the case in long-cultured porcine OECs.

Earlier mentioned FBN1 is also known to interact with another two proteins upregulated in this study, namely GAS6 and TNC, which interact with each other as well (Fig. 5). GAS6 (growth arrest-specific 6) serves as a ligand for AXL receptor tyrosine kinase, activation of which may lead to cell proliferation stimulation [36]. Besides, GAS6 supports platelet macroaggregate formation, with loss of this protein protecting mice against pathological thrombosis, as reported by Angelillo-Scherrer et al. [37]. TNC encodes tenascin $\mathrm{C}$, an extracellular matrix protein typically expressed in tendons and smooth muscle in the adult, as well as in developing cartilage and bone of embryos [38]. Tenascin C has also been reported to participate in mammalian spinal cord development, as it is involved in gliogenic neural precursor cells maturation [39]. Although both GAS6 and TNC are not typically involved in reproductive events, their upregulation after long term OEC cultivation may indicate these cells' differentiation and changing phenotype.
Another upregulated gene was EPAS1, which encodes a HIF2A transcription factor, regulating gene expression under hypoxic conditions. The study conducted by Tian et al. indicates that EPAS1 plays an important role in endothelial cell differentiation, since it is expressed mostly in highly vascularized tissues of adult humans and in endothelial cells of the embryonic and adult mouse [40]. We have also observed a significant increase in NOX4 expression, which encodes the NAD $(\mathrm{P}) \mathrm{H}$ oxidase responsible for reactive oxygen species production. NOX4 is highly expressed in the proximal tubules of renal cortex and has been implicated in oxygen sensing and oxygen-dependent gene expression regulation in kidneys [41]. Similarly to EPAS1, NOX4 is abundant in endothelial cells, with serum removal increasing its expression, resulting in suppression of cell proliferation, as demonstrated by Ago et al. Therefore its involvement in endothelial cell growth and survival has been suggested [42]. It appears that the two aforementioned proteins may be involved in oxygen metabolism in porcine oviductal epithelial cells.

The last of the ten most upregulated genes in our study, $P R L R$, encodes the prolactin receptor, mainly involved in the stimulation of mammary gland development during pregnancy and lactation regulation. PRLR plays crucial role in mammalian reproduction and is expressed in a variety of tissues, including oviductal epithelium in humans and mice [43]. Ormandy et al. demonstrated that mice carrying a germ-line null mutation of PRLR exhibited irregular cycles, reduced fertilization rates, defective preimplantation embryo development and failure of embryonic implantation. The failure of embryo development in the oviduct possibly occurred due to the fact that prolactin stimulates early pregnancy factor (EPF) production and the lack of PRLR results in adverse environment for an embryo [44].

Summing up, our current results revealed a set of genes differentially expressed during porcine OECs long term in vitro cultivation. These genes were assigned to four ontology groups involved in oxygen metabolism, which is crucial for female fertility and proper embryo development. Our findings provide a valuable insight into porcine reproductive biology and may be utilized in optimization of assisted reproductive techniques.

\section{Acknowledgements}

This publication and its results are an outcome of a cooperation between Poznan University of Medical Sciences (Poznań, Poland) and Polish Ministry of Science and Higher Education, with Institute of Advanced Sciences Sp. z o.o. (Poznań, Poland), as a part of the "Professional PhD" programme.

\section{Corresponding author}

Paweł Antosik; Veterinary Centre, Nicolaus Copernicus University in Torun, Torun, Poland; email: pantosik@umk.pl.

\section{Conflict of interest statement}

The authors declare they have no conflicts of interest. 


\section{References}

1. Li S, Winuthayanon W. Oviduct: roles in fertilization and early embryo development. J Endocrinol. 2017;232:R1-26; DOI:10.1530/ JOE-16-0302.

2. Guerin P, El Mouatassim S, Ménézo Y. Oxidative stress and protection against reactive oxygen species in the pre-implantation embryo and its surroundings. Hum Reprod Update. 2001;7:175-89; DOI:10.1093/ humupd/7.2.175.

3. El Mouatassim S, Guérin P, Ménézo Y. Mammalian oviduct and protec tion against free oxygen radicals: Expression of genes encoding antioxidant enzymes in human and mouse. Eur J Obstet Gynecol Reprod Biol. 2000; DOI:10.1016/S0301-2115(99)00169-4

4. Huang VW, Zhao W, Lee CL, Lee CYL, Lam KKW, Ko JKY, Yeung WSB, Ho PC, Chiu PCN. Cell membrane proteins from oviductal epithelial cell line protect human spermatozoa from oxidative damage. Fertil Steril. 2013 DOI:10.1016/j.fertnstert.2012.11.056.

5. Miessen K, Sharbati S, Einspanier R, Schoen J. Modelling the porcine oviduct epithelium: A polarized in vitro system suitable for long-term cultivation. Theriogenology. 2011; DOI:10.1016/j. theriogenology.2011.04.021.

6. Huang DW, Sherman BT, Tan Q, Collins JR, Alvord WG, Roayaei J, Stephens R, Baseler MW, Lane HC, Lempicki RA. The DAVID Gene Functional Classification Tool: a novel biological module-centric algorithm to functionally analyze large gene lists. Genome Biol. 2007;8:R183 DOI:10.1186/gb-2007-8-9-r183.

7. Walter W, Sánchez-Cabo F, Ricote M. GOplot: An R package for visually combining expression data with functional analysis. Bioinformatics. 2015;31:2912-4; DOI:10.1093/bioinformatics/btv300.

8. von Mering C, Jensen LJ, Snel B, Hooper SD, Krupp M, Foglierini M, Jouffre N, Huynen MA, Bork P. STRING: known and predicted protein-protein associations, integrated and transferred across organisms. Nucleic Acids Res. 2004;33:D433-7; DOI:10.1093/nar/gki005.

9. Skowronski MT, Kwon T-H, Nielsen S. Immunolocalization of aquaporin 1,5 , and 9 in the female pig reproductive system. J Histochem Cytochem. 2009;57:61-7; DOI:10.1369/jhc.2008.952499.

10. Skowronski MT, Skowronska A, Nielsen S. Fluctuation of Aquaporin 1, 5, and 9 Expression in the Pig Oviduct during the Estrous Cycle and Early Pregnancy. J Histochem Cytochem. 2011;59:419, DOI:10.1369/0022155411400874.

11. Yin L, Li Y, Ren J, Kuwahara H, Kufe D. Human MUC1 Carcinoma Antigen Regulates Intracellular Oxidant Levels and the Apoptotic Response to Oxidative Stress. J Biol Chem. 2003;278:35458-64; DOI:10.1074/jbc M301987200.

12. Gipson IK, Ho SB, Spurr-Michaud SJ, Tisdale AS, Zhan Q Torlakovic E, Pudney J, Anderson DJ, Toribara NW, Hill JA. Mucin Genes Expressed by Human Female Reproductive Tract Epithelia1. Biol Reprod. 1997;56:999-1011; DOI:10.1095/biolreprod56.4.999.

13. Yamamoto Y, Kohka M, Kobayashi Y, Woclawek-Potocka I, Okuda K. Endothelin as a local regulating factor in the bovine oviduct. Reprod Ferti Dev. 2016;28:673; DOI:10.1071/RD14076

14. An S, Dickens MA, Bleu T, Hallmark OG, Goetzl EJ. Molecular Cloning of the Human Edg2 Protein and Its Identification as a Functional Cellular Receptor for Lysophosphatidic Acid. Biochem Biophys Res Commun 1997;231:619-22; DOI:10.1006/bbrc.1997.6150.

15. Yoshimoto Y, Kobayashi Y, Woclawek-Potocka I, Sinderewicz E, Yamamoto Y, Kimura K, Okuda K. Local effect of lysophosphatidic acid on prostaglandin production in the bovine oviduct. Reprod Fertil Dev. 2016;29:1021; DOI:10.1071/RD15409.

16. Menezes ME, Bhatia S, Bhoopathi P, Das SK, Emdad L, Dasgupta S, Dent P, Wang X-Y, Sarkar D, Fisher PB. MDA-7/IL-24: multifunctional cancer killing cytokine. Adv Exp Med Biol. 2014;818:127-53 DOI:10.1007/978-1-4471-6458-6_6.

17. Sauane M, Su Z-Z, Gupta P, Lebedeva I V, Dent P, Sarkar D, Fisher PB. Autocrine regulation of mda-7/IL-24 mediates cancer-specific apoptosis. Proc Natl Acad Sci U S A. 2008;105:9763-8; DOI:10.1073/ pnas.0804089105.

18. Refaat B, Ahmad J, Idris S, Kamfar FF, Ashshi AM, Batwa SA, Malibary FA. Characterisation of vitamin D-related molecules and calcium-sensing receptor in human Fallopian tube during the menstrual cycle and in ectopic pregnancy. Cell Tissue Res. 2017;368:201-13; DOI:10.1007/ s00441-016-2519-2.

19. Ma R, Gu Y, Zhao S, Sun J, Groome LJ, Wang Y. Expressions of vitamin D metabolic components VDBP, CYP2R1, CYP27B1, CYP24A1, and VDR in placentas from normal and preeclamptic pregnancies. Am J Physiol Endocrinol Metab. 2012;303:E928-35; DOI:10.1152/ajpendo.00279.2012.

20. Cobaleda C, Pérez-Caro M, Vicente-Dueñas C, Sánchez-García I. Function of the Zinc-Finger Transcription Factor SNAI2 in Cancer and De- velopment. Annu Rev Genet. 2007;41:41-61; DOI:10.1146/annurev. genet.41.110306.130146.

21. Fedorova L, Gatto-Weis C, Smaili S, Khurshid N, Shapiro JI, Malhotra D, Horrigan T. Down-regulation of the transcription factor snail in the placentas of patients with preeclampsia and in a rat model of preeclampsia. Reprod Biol Endocrinol. 2012;10:15; DOI:10.1186/1477-7827-10-15.

22. Shields JM, Christy RJ, Yang VW. Identification and characterization of a gene encoding a gut-enriched Krüppel-like factor expressed during growth arrest. J Biol Chem. 1996;271:20009-17; DOI:10.1074/ jbc.271.33.20009.

23. Xu L, Sun H, Zhang M, Jiang Y, Zhang C, Zhou J, Ding L, Hu Y, Yan G. MicroRNA-145 protects follicular granulosa cells against oxidative stress-induced apoptosis by targeting Krüppel-like factor 4. Mol Cell Endocrinol. 2017;452:138-47; DOI:10.1016/j.mce.2017.05.030.

24. Amson R, Sigaux F, Przedborski S, Flandrin G, Givol D, Telerman A. The human protooncogene product p33pim is expressed durin fetal hematopoiesis and in diverse leukemias. Proc Natl Acad Sci. 1989;86:8857-61; DOI:10.1073/pnas.86.22.8857.

25. Ngo JK, Pomatto LCD, Davies KJA. Upregulation of the mitochondrial Lon Protease allows adaptation to acute oxidative stress but dysregulation is associated with chronic stress, disease, and aging. Redox Biol. 2013;1:258-64; DOI:10.1016/j.redox.2013.01.015

26. Sun XJ, Rothenberg P, Kahn CR, Backer JM, Araki E, Wilden PA, Cahill DA, Goldstein BJ, White MF. Structure of the insulin receptor substrate IRS-1 defines a unique signal transduction protein. Nature. 1991;352:73-7; DOI:10.1038/352073a0

27. Puscheck EE, Pergament E, Patel Y, Dreschler J, Rappolee DA. Insulin receptor substrate- 1 is expressed at high levels in all cells of the peri-implantation mouse embryo. Mol Reprod Dev. 1998;49:386-93; DOI:10.1002/ (SICI)1098-2795(199804)49:4<386::AID-MRD5>3.0.C0;2-J.

28. Lawn RM, Wade DP, Garvin MR, Wang X, Schwartz K, Porter JG, Seilhamer JJ, Vaughan AM, Oram JF. The Tangier disease gene product ABC1 controls the cellular apolipoprotein-mediated lipid removal pathway. J Clin Invest. 1999;104:R25-31; DOI:10.1172/JCI8119.

29. Langmann T, Klucken J, Reil M, Liebisch G, Luciani M-F, Chimini G, Kaminski WE, Schmitz G. Molecular Cloning of the Human ATP-Binding Cassette Transporter 1 (hABC1): Evidence for Sterol-Dependent Regulation in Macrophages. Biochem Biophys Res Commun. 1999;257:29-33; DOI:10.1006/bbrc.1999.0406.

30. Emanuel BS, Cannizzaro LA, Seyer JM, Myers JC. Human alpha 1(III) and alpha 2(V) procollagen genes are located on the long arm of chromosome 2. Proc Natl Acad Sci. 1985;82:3385-9; DOI:10.1073/pnas.82.10.3385.

31. Andrikopoulos K, Liu X, Keene DR, Jaenisch R, Ramirez F. Targeted mutation in the col5a2 gene reveals a regulatory role for type $\mathrm{V}$ collagen during matrix assembly. Nat Genet. 1995;9:31-6; DOI:10.1038/ng0195-31.

32. Sakai LY, Keene DR, Engvall E. Fibrillin, a new 350-kD glycoprotein, is a component of extracellular microfibrils. J Cell Biol. 1986;103:2499509; DOI:10.1083/jcb.103.6.2499.

33. Wu X, Li H, Kang L, Li L, Wang W, Shan B. Activated Matrix Metalloproteinase-2-A Potential Marker of Prognosis for Epithelial Ovarian Can cer. Gynecol Oncol. 2002;84:126-34; DOI:10.1006/GYN0.2001.6477.

34. Roberts LM, Visser JA, Ingraham HA. Involvement of a matrix metalloproteinase in MIS-induced cell death during urogenital development. Development. 2002;129:1487-96.

35. Chen P-S, Zhai W-R, Zhou X-M, Zhang J-S, Zhang Y-E, Ling Y-Q, Gu Y-H. Effects of hypoxia, hyperoxia on the regulation of expression and activity of matrix metalloproteinase-2 in hepatic stellate cells. World J Gastroenterol. 2001;7:647; DOI:10.3748/wjg.v7.i5.647.

36. Varnum BC, Young C, Elliott G, Garcia A, Bartley TD, Fridell Y-W, Hunt RW, Trail G, Clogston C, Toso RJ, Yanagihara D, Bennett L, Sylber M, Merewether LA, Tseng A, Escobar E, Liu ET, Yamane HK. Axl receptor tyrosine kinase stimulated by the vitamin K-dependent protein encoded by growth-arrest-specific gene 6. Nature. 1995;373:623-6; DOI:10.1038/373623a0.

37. Angelillo-Scherrer A, Burnier L, Flores N, Savi P, DeMol M, Schaeffer P, Herbert J-M, Lemke G, Goff SP, Matsushima GK, Earp HS, Vesin C, Hoylaerts MF, Plaisance S, Collen D, Conway EM, Wehrle-Haller B, Carmeliet P. Role of Gas6 receptors in platelet signaling during thrombus stabilization and implications for antithrombotic therapy. J Clin Invest. 2005;115:237-46; DOI:10.1172/JCI22079.

38. Pearson CA, Pearson D, Shibahara S, Hofsteenge J, Chiquet-Ehrismann R. Tenascin: cDNA cloning and induction by TGF-beta. EMBO J. 1988;7:2977-82.

39. Karus M, Denecke B, ffrench-Constant C, Wiese S, Faissner A. The extracellular matrix molecule tenascin $\mathrm{C}$ modulates expression levels and territories of key patterning genes during spinal cord astrocyte specification. Development. 2011;138:5321-31; DOI:10.1242/dev.067413. 
40. Tian H, McKnight SL, Russell DW. Endothelial PAS domain protein 1 (EPAS1), a transcription factor selectively expressed in endothelial cells. Genes Dev. 1997;11:72-82; DOI:10.1101/gad.11.1.72.

41. Geiszt M, Kopp JB, Várnai P, Leto TL. Identification of renox, an NA D(P)H oxidase in kidney. Proc Natl Acad Sci U S A. 2000;97:8010-4; DOI:10.1073/pnas.130135897.

42. Ago T, Kitazono T, Ooboshi H, Iyama T, Han YH, Takada J, Wakisaka M, Ibayashi S, Utsumi H, Iida M. Nox4 as the Major Catalytic Component of an Endothelial NAD(P)H Oxidase. Circulation. 2004;109:227-33; DOI:10.1161/01.CIR.0000105680.92873.70.

43. Shao R, Nutu M, Weijdegård B, Egecioglu E, Fernandez-Rodriguez J, Tallet E, Goffin V, Ling C, Billig H. Differences in Prolactin Receptor (PRLR) in Mouse and Human Fallopian Tubes: Evidence for Multiple Regulatory Mechanisms Controlling PRLR Isoform Expression in Mice1. Biol Reprod. 2008;79:748-57; DOI:10.1095/biolreprod.108.070003.

44. Ormandy CJ, Camus A, Barra J, Damotte D, Lucas B, Buteau H, Edery M, Brousse N, Babinet C, Binart N, Kelly PA. Null mutation of the prolactin receptor gene produces multiple reproductive defects in the mouse. Genes Dev. 1997;11:167-78; DOI:10.1101/gad.11.2.167. 